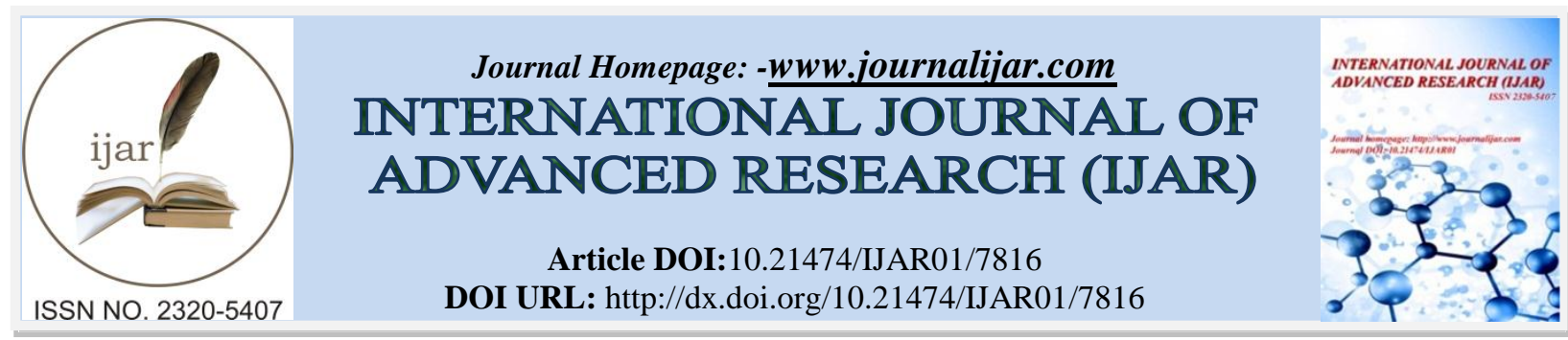

RESEARCH ARTICLE

\title{
POTENTIAL IMPACT OF CULTURE MEDIUM REFRESH AT DAY 3 ON DEVELOPMENT AND QUALITY OF EMBRYOS IN ICSI TREATMENT CYCLES.
}

\section{Azza A. Abdel Razek ${ }^{1}$, Soaad A. Noaman ${ }^{1}$, Hazem M. EL-Hariri ${ }^{3}$, Gamal F. Mustafa ${ }^{1}$ and Omaima A.} Khamiss'.

1. Department of obstetrics and gynecology, Ain Shams University, Egypt.

2. Department of Animal biotechnologY, Genetic Engineering and BiotechnologY Research Institute (GEBRI), Sadat City University, Egypt.

3. Department of Community, environmental \& industrial health, Ain Shams University, Egypt.

\section{Manuscript Info}

\section{Manuscript History}

Received: 6 August 2018

Final Accepted: 8 September 2018

Published: October 2018

Keywords:-

ICSI, Embryo culture, single medium, uninterrupted culture, with and without Renewal media..

\section{Abstract}

Background: The introduction of Intracytoplasmic Sperm Injection (ICSI) in 1992, followed by reports of high success rates using this method, has opened a new era in the treatment of previously untreatable cases of infertility especially male factor infertility. Embryo culture refers to the culture of human gametes and embryos during in vitro fertilization treatments. The procedure is initiated when eggs and sperm are combined in a culture dish and it ends with the transfer of the resulting embryo to the uterus. The culture period varies between one and six days, and the embryo culture process is vital to the success of any IVF or ICSI procedure. The type of culture medium used may affect embryo quality and hence the success rate of the treatment.

Aim of the Study: To evaluate the potential impact of culture medium refresh on day 3 on the development and quality of embryos during in vitro fertilization in ICSI treatment cycles.

Patients and Methods: This Randomized Comparative Prospective Controlled Trail study was conducted on 200 patients(corresponding to 763 day 3 embryos in each group) undergoing ICSI at Assisted Reproduction unit - Ain Shams University Maternity Hospital Starting from October of 2015 to October of 2017. At day 3 Cleavage stage, patients were divided to two groups according to randomized table. Group I: embryos were refreshed with pre incubated single culture media Global ${ }^{\circledR}$ total media for at least $8 \mathrm{hr}$, extending to day 5 Blastocyst stage. Group II: embryos cultures were continued with the same culture media extending to day 5 without refreshment.

Results: Significant differences in pregnancy rates and implantation rates were not observed between the two groups; but there is significant difference in blastocyst formation were observed, in day-3 Grade-I embryos, improvement was significantly more frequent among renewal group, stability of embryo condition was non-significantly different between renewal and non-renewal media groups, while deterioration of grade was significantly more frequent among non-renewal group. 
Conclusion: In spite of a statistically significant difference was found between the groups in the blastocyst formation and quality of formed blastocyst a non-significant trend was observed between the two groups in implantation and pregnancy rates

Copy Right, IJAR, 2018,. All rights reserved.

\section{Introduction:-}

Beginning in the early 1970s, work utilizing assisted reproductive technology (ART) to treat human infertility intensified. The credit goes to Robert Edwards and Patrick Steptoe for successfully integrating ART in human reproduction, which, in 1978, yielded the birth of the world's first so-called "test-tube baby" Louise Brown (Wang and Sauer, 2006). Given the documented sensitivities of human gametes and embryos to several chemical and physical factors within the human IVF laboratory, ensuring that each human IVF laboratory has an adequate quality control and assurance program in place is a prerequisite for optimizing performance and the successful maintenance of pregnancy outcome. Unless one can guarantee the quality of each component of the culture system (Gardner and Lane, 2003), and can track each lot number of media and consumables, then it is extremely difficult to maintain laboratory performance (Mortimer and Mortimer, 2015). Embryo culture refers to the culture of human gametes and embryos during in vitro fertilization treatments. The procedure is initiated when eggs and sperm are combined in a culture dish and it ends with the transfer of the resulting embryo to the uterus. The culture period varies between one and six days, and the embryo culture process is vital to the success of any IVF or ICSI procedure. The type of culture medium used may affect embryo quality and hence the success rate of the treatment (Youssef et al., 2015). Discussions arising from publications on the effects of human embryo culture media on resultant birth weights need to be tempered in light of the fact that so many variables can impact transfer outcome.

Establishment of an optimal culture system is critical for the correct development of human embryos in vitro and the success of assisted reproduction treatments. In recent years, considerable progress has been made toward the improvement of the existing culture media and the development of a new generation of incubators that are better adapted to the needs of the in vitro fertilization (IVF) field. Currently, human embryos can be cultured in vitro up to the blastocyst stage with relatively high success rates in either single or sequential media (Nunocosta-Broges et al.,2016).

Selecting the best embryo to transfer is a crucial step in in vitro fertilization (IVF) cycles. Before reaching this point, the selected embryos go through a series of events that might compromise their development and viability. One of the most remarkable is the days spent in culture medium environment (Wintner et al., 2017).

The movement of embryos to new dishes, or refreshing the culture medium at intervals has been suggested as a technique to avoid exposure of embryos to the potential buildup of ammonium from the breakdown of amino acids or volatile atmospheric compounds. Single media (sometimes referred to as monoculture media) are designed to provide all components to embryos at all times; a modern single-culture medium, designed to limit the buildup of ammonium by replacement of glutamine with a more stable form, could be used for continuous uninterrupted culture of human embryos, as has been suggested and demonstrated by others (Huisman, 2000-Biggers, 2002-Macklon, 2002 and Biggers, 2008).

\section{Subjects and Methods:-}

Randomized Comparative Prospective Controlled Trail conducted on 200 patients (corresponding to 763 day 3 embryos in each group) ( undergoing ICSI at Assisted Reproduction unit - Ain Shams University Maternity Hospital Starting from October, 2015 to October, 2017.

\section{Randomized and Concealed Allocation}

Random allocation was performed using a computer -generated randomization system .The allocated group numbers are concealed in sealed opaque serially-numbered envelops which are kept with one of the supervisors and are only opened after recruitment allocated patient. 


\section{Sample size justification}

Depending on (LAURENCE BISSONNETTE M.SC. et al,2011 ) who found that Blastulation rate at day 5 (38.3\% versus $22.4 \%$, respectively) in renewal and non-renewal groups , and assuming the power $=0.80$ and $\alpha=0.05$, and by using PASS 11th release the minimal sample size for an equal controlled is 132 day-3 embryos in each group. We will recruit 200 patients (corresponding to 763 day 3 embryos in each group)

\section{The aim of the study}

To assess impact of culture medium refresh on day 3 on development and quality of embryos in ICSI treatment cycles.

\section{Settings:-}

Population: 200 patients (corresponding to 763 day 3 embryos in each group) undergoing ICSI at Assisted Reproduction unit - Ain Shams University Maternity Hospital Starting from October, 2015 to October, 2017.

\section{Subjects Recruitment}

After the initial evaluation of the infertile couple, exclusion and inclusion criteria were applied. Each couple whose embryos were enrolled in the study were invited to participate, adequately counseled and signed a written informed consent in addition to the regulatory and official consents taken in the ICSI protocols.

\section{Research Methodology:}

All patients were subjected to the following:-

1. Pre-enrollment assessment as per unit protocol

2. Controlled ovarian hyperstimulation and intracytoplasmic sperm injection - embryo transfer cycle.

\section{Treatment Plan}

The standard protocol applied was long protocol to all patients under the study according to assisted reproduction unit policy. Patients started combined oral contraceptive Pills on first day of Menses then GnRH analogues (Decapeptyl $0.1 \mathrm{mg}$-ferring pharmaceuticals, Germany) was started on day seventeen of cycle and continued till HMG was started on day two or three of next cycle for ovarian stimulation. Following Confirmation of Pituitary desensitization, defined as an endometrial thickness of $<5 \mathrm{~mm}$ and no ovarian activity evident on transvaginal ultrasound in association with an estradiol concentration $<50 \mathrm{pg} / \mathrm{ml}$. First, follicullometry was done on day six of starting HMG and repeated every other day till the size of follicles reached $14 \mathrm{~mm}$ then it was repeated daily till reaching the condition of having at least three follicles $18 \mathrm{~mm}$ in size at which point the trigger with hCG was given at dose of 10,000IU (Choriomon 5000IU.IBSA.switherland) preparing for Ovum Pick up, trans vaginal ultrasound guided oocyte retrieval will be performed $34-36 \mathrm{hr}$. after hCG administration.

\section{Oocytes collection}

Retrieval of Oocytes was performed under transvaginal ultrasound guidance (Mindray, DP-8800 Plus, china). The aspirates were collected in pre-warmed tubes, and then oocytes were washed in prewarmed Global with HEPES buffered medium.

\section{Denudation and assessment}

The oocytes denudation was done under stereomicroscope (Zeiss stemi2000, Oberkoche, Germany with high magnification. and assessment of maturity was undertaken by inverted microscope (Nikon eclipse TE 2000S,Tokyo,Japan). The nuclear status of denuded oocytes was subsequently recorded as Germinal vesicle (GV), Metaphase I (MI), Metaphase II (MII).

\section{Oocyte Maturational Status}

Maturational status of each oocyte at retrieval was determined by morphology assessment using inverted microscope, the overall appearance of the cytoplasm, zona pullicida and polar body, as well as the granulosa, cumulus, and corona companion somatic cells are considered.

\section{Semen processing before ICSI}

Semen sample were collected by masturbation after 2-7 days of abstinence. the preparation for ICSI was performed after complete liquefactions, the semen was washed with tissue culture media, and final pellet was re suspended in $0.2 \mathrm{ml}$ of sperm washing medium,(Irvine Scientific, Santa Ana,USA) until time of injection (WHO,2010). 


\section{Microinjection}

Under Nikon Eclipse TE 2000-s occupied by Hoffman Modulation contrast, with the attached micromanipulator Integra -1(RI, Cornwell, Uk), microinjection took place.

\section{Embryo culture and development}

Injected oocytes were cultured in Global @ total single culture medium with ovoil@overly in the falcon dishes, each inseminated oocyte incubated in one drop and put inside Triple gas incubators (labotect ® C200, Gottingen, Germany) adjusted to $37.0 \mathrm{c}, 6 \% \mathrm{CO}^{2}$ and $5 \% \mathrm{O}_{2}$ to be visualized after $16-18 \mathrm{hrs}$ to check fertilization.

\section{Assessment of Fertilization and cleavage stage embryo}

Fertilization was assessed 18-20 hrs. from insemination /injection and confirmed by presence of 2 pronuclei (2PN) and the alignment of nuclear precursor bodies (NPB). At the same time, the pronuclear morphological score was assessed. The observation of $2 \mathrm{PN}$ was performed using inverted microscope with Hoffman modulation contrast at X400 magnification (TE 2000 U, Nikon crop. USA). Zygotes were evaluated according to (Alpha Scientists in reproductive Medicine and ESHRE Special Interest Group of Embryology, 2011).

At day 3 Cleavage stage, patients were divided to two groups according to randomized table:

1. Group I: culture media were refreshed with pre incubated culture media Global ${ }^{\circledR}$ total media for at least $8 \mathrm{hr}$ and extending to day 5 Blastocyst stage.

2. Group II: no culture refreshment was done, embryos kept in the same culture media and continued cultured to day 5 with the same culture media.

\section{Assessment of Day 5 Embryo (Blastocyst).}

Similar to the cleavage stage embryos, embryo morphological features are very important in assessment of blastocyst quality and its reproductive potential. Assement was done according to blastocyst scoring system was proposed by Gardner (Gardner et al.,2000).

\section{Embryo transfer (ET)}

The best quality embryo- maximum 2 embryos were transferred at day 5. Embryo transfer done guided by trans abdominal ultrasound in all cases.

\section{Luteal phase support}

Medication is prescribed for patient by oral, vaginal and intramuscular progesterone. After 9 day from embryo transfer serum Human chorionic gonadotrophins (HCG) measured, if positive test detected patient followed up with transvaginal ultrasound after 20 days for clinical pregnancy detection.

\section{Statistical methods}

The collected data were coded, tabulated, and statistically analyzed using IBM SPSS statistics (Statistical Package for Social Sciences) software version 22.0, IBM Corp., Chicago, USA, 2013.

Descriptive statistics were done for quantitative data as minimum\& maximum of the range as well as mean $\pm \mathrm{SD}$ (standard deviation)for quantitative normally distributed data, median and, while it was done for qualitative data as number and percentage.

Inferential analyses were done for quantitative variables using independent t-test in cases of two independent groups with normally distributed data and paired t-test in cases of two dependent groups with normally distributed data. In qualitative data, inferential analyses for independent variables were done using Chi square test for differences between proportions and Fisher's Exact test for variables with small expected numbers. The level of significance was taken at $P$ value $<0.050$ is significant, otherwise is non-significant.

\section{Results:-}

In this study a total of 200 infertile couple underwent ICSI Cycle treatment divided to two study groups using randomized table. In media refresh group $835 \mathrm{MII}$ oocytes were cultured via the two step protocol, meaning that embryos were cultured from day 0 to day 3 in individual wells prepared with single-step medium then were moved, maintaining their individuality, to a new Embryo Slide previously prepared and equilibrated with fresh medium to follow their development from day 3 up to day 5 , their fertilization rate were $91.4 \%$. 
In Non culture media refresh group 969 MII were cultured from day 0 up to day 5 without renewal or refreshment of the culture medium on day 3 , hence the embryos were not manipulated and the culture conditions were not disturbed during the entire in vitro culture period up to D5, their fertilization rate was 91.6\%. Data showed no statistically significant difference between the two studied groups regarding fertilization and cleavage Rates.

Table 1:- Fertilization and Cleavage rates among the studied groups.

\begin{tabular}{|l|c|c|c|}
\hline \multicolumn{1}{|c|}{ Outcome } & Refreshed & Non-refreshed & P \\
\hline \multicolumn{1}{|c|}{ Fertilization outcome } & $(\mathbf{N = 8 3 5 )}$ & $\mathbf{( N = 9 6 9 )}$ & $\# 0.841$ \\
\hline $\begin{array}{l}\text { Fertilization Rate } \\
=\text { Fertilized*100/MII }\end{array}$ & $763(91.4 \%)$ & $888(91.6 \%)$ & $\# 0.208$ \\
\hline \multicolumn{1}{|c|}{ Cleavage outcome } & $(\mathbf{N}=\mathbf{7 6 3})$ & $\mathbf{( N = 8 8 8 )}$ & \\
\hline $\begin{array}{l}\text { Cleavage Rate } \\
=\text { Cleaved*100/Fertilized }\end{array}$ & $730(95.7 \%)$ & $860(96.8 \%)$ & \\
\hline
\end{tabular}

\#Chi square test

The blastocyst development rates $(69.2 \%$ vs. $61.6 \%$; $\# 0.002 *)$ and good-quality blastocyst rates (87.1\% vs. $78.7 \%$; P\#<0.001*) were found statistically significantly different between the two-step and one-step culture groups.

Table (2): Comparison of development blastocyst and Good Quality Blastocyst rate between study groups

\begin{tabular}{|c|c|c|c|c|}
\hline Group & $\begin{array}{c}\text { Number of } \\
\text { embryos } \\
\text { (day-3) } \\
\text { (Total sum) }\end{array}$ & $\begin{array}{c}\text { Number of blastocyst } \\
\text { development } \% \\
(\text { day-5) } \\
(\% \text { from day-3) }\end{array}$ & $\begin{array}{c}\text { Good quality blastocyst } \\
\text { (Grade-I/II) } \\
\text { Total (\%) } \\
(\% \text { from day-5) }\end{array}$ & $\begin{array}{c}\text { Good } \\
\text { quality } \\
\text { blastocys } \\
\text { t } \\
\text { transferr } \\
\text { ed }(\%) \\
(\% \text { from } \\
\text { day-5) } \\
\end{array}$ \\
\hline Refreshed & 730 & $505(69.2 \%)$ & $\begin{array}{c}440(87.1 \%) \\
\quad(N=505)\end{array}$ & $\begin{array}{c}184 \\
(36.4 \%) \\
(\mathrm{N}=505)\end{array}$ \\
\hline $\begin{array}{c}\text { Non- } \\
\text { refreshed }\end{array}$ & 860 & $530(61.6 \%)$ & $\begin{array}{c}417(78.7 \%) \\
\quad(\mathrm{N}=530)\end{array}$ & $\begin{array}{c}183 \\
(34.5 \%) \\
(\mathrm{N}=530)\end{array}$ \\
\hline $\mathbf{P}$ & & $\# 0.002 *$ & $\#<0.001 *$ & $\# 0.521$ \\
\hline
\end{tabular}

\#Chi square test, *Significant

Table 3:-Comparison between embryo grading in day-3 in both study groups.

\begin{tabular}{|c|c|c|c|c|}
\hline Outcome & & Refreshed & Non-refreshed & $\mathbf{P}$ \\
\hline \multirow[t]{4}{*}{ Grade-I } & Total & 575 & 568 & \\
\hline & Improved & $312(54.3 \%)$ & $252(44.4 \%)$ & $\#<0.001 *$ \\
\hline & Stable & $74(12.9 \%)$ & $88(15.5 \%)$ & $\# 0.204$ \\
\hline & Deteriorated & $189(32.9 \%)$ & $228(40.1 \%)$ & \#0.011* \\
\hline \multirow[t]{4}{*}{ Grade-II } & Total & 92 & 167 & \\
\hline & Improved & $10(10.9 \%)$ & $22(13.2 \%)$ & $\# 0.590$ \\
\hline & Stable & $41(44.6 \%)$ & $50(29.9 \%)$ & \#0.018* \\
\hline & Deteriorated & $41(44.6 \%)$ & $95(56.9 \%)$ & \#0.057 \\
\hline \multirow[t]{3}{*}{ Grade-III } & Total & 63 & 125 & \multirow[t]{3}{*}{$\$ 1.000$} \\
\hline & Improved & $3(4.8 \%)$ & $5(4.0 \%)$ & \\
\hline & Stable & $60(95.2 \%)$ & $120(96.0 \%)$ & \\
\hline
\end{tabular}

\#Chi square test, §Fisher's Exact test, *Significant

In day-3 Grade-I embryos, improvement (Change from day-3 grade-II to day-5 grade-I and/or persistence of day-3 grade-I as day-5 grade-I) was significantly more frequent among refreshed group, stable (persistence of day-3 gradeII as day-5 grade-II and/or persistence of day-3 grade-III as day-5 grade-III) was non-significantly different among refreshed and non-refreshed groups, while deterioration (Change from day-3 grade-I to day-5 grade-II and/or change 
from day-3 grade-II to day-5 grade-III and/or change from day-3 grade-III to day-5 arrest) was significantly more frequent among non-refreshed group.

In day-3 Grade-II embryos, improvement (Change from day-3 grade-II to day-5 grade-I and/or persistence of day-3 grade-I as day-5 grade-I) was non-significantly different among refreshed group and non-refreshed group, while stable (persistence of day-3 grade-II as day-5 grade-II and/or persistence of day-3 grade-III as day-5 grade-III) was significantly more frequent among refreshed group while deterioration (Change from day-3 grade-I to day-5 grade-II and/or change from day-3 grade-II to day-5 grade-III and/or change from day-3 grade-III to day-5 arrest) was significantly more frequent among non-refreshed group.

In day-3 Grade-III embryos, no significant difference between refreshed and non-refreshed media regarding changes.

Table 4:-Comparison between refreshed and non-refreshed media regarding changes from day-3 to day-5 in embryo quality

\begin{tabular}{|c|l|c|c|}
\hline Outcome & & Day-3 & Day-5 \\
\hline \multirow{4}{*}{ Refreshed media } & \multicolumn{1}{|c|}{ Total } & 730 & 730 \\
\cline { 2 - 4 } & Grade-I & $575(78.8 \%)$ & $325(44.5 \%)$ \\
\cline { 2 - 4 } & Grade-II & $92(12.6 \%)$ & $115(15.8 \%)$ \\
\cline { 2 - 4 } & Grade-III & $63(8.6 \%)$ & $65(8.9 \%)$ \\
\cline { 2 - 4 } & Arrest & $0(0.0 \%)$ & $225(30.8 \%)$ \\
\hline \multirow{5}{*}{ Non-refreshed media } & & 860 & 860 \\
\cline { 2 - 5 } & Grade-I 568(66.0\%) & $276(32.1 \%)$ \\
\cline { 2 - 5 } & Grade-II & $167(19.4 \%)$ & $141(16.4 \%)$ \\
\cline { 2 - 5 } & Grade-III & $125(14.5 \%)$ & $113(13.1 \%)$ \\
\cline { 2 - 5 } & Arrest & $0(0.0 \%)$ & $330(38.4 \%)$ \\
\hline
\end{tabular}

Of a total of 385 embryos were transferred to 200 patients, Chemical pregnancy was (64\%), Clinical pregnancy $(53 \%)$, implantation rate $(36.8 \%)$ \& Live birth $(53 \%)$ in refreshed group. In non refreshed group; chemical pregnancy was $(61.0 \%)$, clinical pregnancy $(52.0 \%)$, implantation rate $(23.3 \%) \&$ Live birth (52.0\%). No significant difference between the studied groups regarding embryo transfer number, chemical \& clinical pregnancies, implantation rate and live births rates.

Table 5:-Comparison of the clinical outcomes between two Study Groups

\begin{tabular}{|l|c|c|c|}
\hline \multicolumn{1}{|c|}{ Outcome } & Refreshed & Non-refreshed & P \\
\hline No of patients & $\mathbf{1 0 0}$ & $\mathbf{1 0 0}$ & $\wedge$ \\
\hline Average of embryo transferred & $1.9 \pm 0.3$ & $2.0 \pm 0.2$ & \\
\hline Number of patients transfers & $\mathbf{1 0 0}$ & $\mathbf{1 0 0}$ & \\
\hline Number of embryo transferred & $\mathbf{1 9 0}$ & $\mathbf{1 9 5}$ & $\# 0.661$ \\
\hline Positive $\boldsymbol{\beta}$-HCG (\%) & $64(64.0 \%)$ & $61(61.0 \%)$ & $\# 0.887$ \\
\hline Clinical pregnancies (\%) & $53(53.0 \%)$ & $52(53.0 \%)$ & $\# 0.350$ \\
\hline Implantation Rate\% & $70(36.8 \%)$ & $63(32.3 \%)$ & \\
No. of embryos US $* \mathbf{1 0 0} /$ Transferred & & & \\
\hline Live birth $(\%)$ & $53(53.0 \%)$ & $52(52.0 \%)$ & $\# 0.887$ \\
\hline
\end{tabular}

${ }^{\wedge}$ Independent t-test, \#Chi square test.

In refreshed group Good Quality embryo at day 3 were (91.4)\%, Blastocyst Formation (69.2\%) \& good Quality Blastocyst Formed (60.3\%) while, Good Quality embryo at day 3 were (85.5)\%, Blastocyst Formation (61.6\%) \& good Quality Blastocyst Formed (48.5\%) in non refresh group which mean, Blastocyst formation and good quality Blastocyst were significantly higher among refreshed group. 
Table 6:-Comparison of Good Quality embryo at day 3,Blastocyst Formation \&Good Quality Blastocyst between Both Study groups.

\begin{tabular}{|l|c|c|c|}
\hline \multicolumn{1}{|c|}{ Outcome } & $\begin{array}{c}\text { Refreshed } \\
(\mathbf{N}=\mathbf{7 3 0})\end{array}$ & $\begin{array}{c}\text { Non-refreshed } \\
(\mathbf{N = 8 6 0 )}\end{array}$ & P \\
\hline Good quality embryo (day-3) & $667(91.4 \%)$ & $735(85.5 \%)$ & $\#<\mathbf{0 . 0 0 1 *}$ \\
\hline $\begin{array}{l}\text { Blastocyst formation (total) } \\
\text { (day-5) }\end{array}$ & $505(69.2 \%)$ & $530(61.6 \%)$ & $\# \mathbf{0 . 0 0 2 *}$ \\
\hline Good quality Blastocyst (I/II) & $440(60.3 \%)$ & $416(48.5 \%)$ & $\#<\mathbf{0 . 0 0 1 *}$ \\
\hline
\end{tabular}

\#Chi square test, §Fisher's Exact test, *Significant

There were no significant difference between the studied groups regarding the overall mean of gestational age and number of gestational sacs for two study groups.

Table 7:Comparison of Gestational age and gestational Sacs between both study groups.

\begin{tabular}{|c|c|c|c|c|}
\hline Outcome & & $\begin{array}{l}\text { Refreshed } \\
(\mathrm{N}=52)\end{array}$ & $\begin{array}{c}\text { Non-refreshed } \\
(\mathrm{N}=52)\end{array}$ & $P$ \\
\hline \multirow[t]{3}{*}{ Number of Gestational Sacs } & Singleton (n) & $39(73.6 \%)$ & $42(80.8 \%)$ & \multirow[t]{3}{*}{$\$ 0.510$} \\
\hline & Twin (n) & $11(20.8 \%)$ & $9(17.3 \%)$ & \\
\hline & Triplet (n) & $3(5.7 \%)$ & $1(1.9 \%)$ & \\
\hline \multirow[t]{2}{*}{ GA at delivery } & Full term & $51(96.2 \%)$ & $49(94.2 \%)$ & \multirow[t]{2}{*}{$\$ 0.678$} \\
\hline & Preterm & $2(3.8 \%)$ & $3(5.8 \%)$ & \\
\hline
\end{tabular}

$\S$ Fisher's Exact test

\section{Discussion:-}

Culture media are generally considered to play an important role in the success rates of an assisted reproductive technology (ART) program (Mantikou 2013). They could have an effect on the health of the newborns (Dumoulin, 2010).

Earlier studies have shown that the environment of gametes and pre-implantation embryos has an effect on the expression and imprinting of a number of key genes that affect the development of the embryos (Blake 2007; Fauque 2007). The differences in success rates of IVF treatments could be explained by differences in the composition and balance of the elements present in culture medium. Currently, there is limited information concerning the composition of the culture media, which restricts the understanding of the mechanism of action.

In a recent study, Kleijkers et al. (2015) sought to determine whether gene expression in human preimplantation embryos is affected by the medium used for embryo culture in vitro during IVF treatment. Other factors during embryo culture could influence IVF/ICSI success rates. Such factors include the number of embryos per drop and culture dish and embryo produced factors (Hoelker et al., 2010).

Although various embryo culture conditions, such as culture medium, embryo density, supplementation of growth factors, and $\mathrm{O}_{2}$ concentration, have been extensively investigated and optimized (Jiao GZ et al.,2016), quality of embryos produced in vitro is still markedly lower, suggesting the involvement of other unknown factors that may be crucial for further optimization and improvement.

Replacing the culture medium is a routine step for in vitro culture (IVC) of bovine embryos. This process might have positive effects on the development of embryos by supplying some necessary nutrients for development and removing toxic metabolites, such as ammonia and free oxygen radicals accumulated in the culture medium during IVC (Hashimoto S et al.,2008).

Continual culture in the presence of amino acids (especially glutamine) could reduce the viability of embryos as a consequence of ammonia formation resulting from spontaneous degradation of amino acids and/or their catabolism by the embryo (Gardner DK, Lane M,1993).

In reality, the risk of ammonia toxicity is very low. Glutamine degradation is very weak in alkaline $\mathrm{pH}$ and less than $1 \%$ of the ammonia formed in culture medium is retained. The salts formed, ammonium carbonate $\left(\mathrm{NH}_{4}\right)_{2} \mathrm{CO}_{3}$ and bicarbonate $\left(\mathrm{NH}_{4} \mathrm{HCO}_{3}\right)$ are highly unstable in a slightly alkaline $\mathrm{pH}$, as in classical in vitro culture conditions. Their 
$\mathrm{PKa}$ is 7.2 at $25^{\circ} \mathrm{C}$, and $\mathrm{NH}_{3}$ is released in the $\mathrm{CO}_{2}$ flux. Moreover, ammonia can also be removed by transamination of pyruvate to alanine. Alanine is then released into the culture medium (Brison DR et al.,2004), probably indicating an original dynamic system for removal of ammonia. The possibility that an ammonium gradient might be transitorily formed when embryo culture is performed in very small microdrops under oil cannot be excluded.

Adding $\mathrm{NH}_{4} \mathrm{Cl}$ to culture media has recently been proposed as a model to mimic the toxicity of ammonium ions. However, $\mathrm{NH}_{4} \mathrm{Cl}$ is a highly stable acidic salt: this model does not reflect the physiological reality, and is therefore invalid. In fine, this poorly established concept has led to a reduction in the concentration of amino acids in embryo culture media relative to the in vivo environment (Elhassan YM et al.,2001)thus decreasing their "osmolyteprotecting role" towards the embryo.

As a consequence, osmolarity is decreased to 260 milli-osmoles in some commercial culture media, in order to counteract a possible negative effect of low amino acid concentrations (vide supra Physico-chemical parameters, electrolytes and embryo metabolism). However, this decreased amino acid concentration in culture media is likely to have a negative impact on some important embryo physiological processes such as imprinting (Hiura $\mathrm{H}$ et al.,2012).

However, renewal of culture medium also causes an abrupt change in the microenvironment. these sud.den changes of physical and chemical properties may result in stress to cultured embryos (Biggers JD, Racowsky,2002).

The present study focused on the impact of renewing the embryo culture media on day 3 on development ant quality of embryo, this study was conducted on 200 ICSI cycle, IVF laboratories performing these procedures must comply with strict requirements to control the conditions to which embryo cultures are exposed, including temperature variations, light, and $\mathrm{O}_{2}$ and $\mathrm{CO}_{2}$ pressure (Cohen et al., 1997). It is also important to control for substances that might affect the development of the embryos, such as dust particles, VOC, and disinfectants.

(Costa-Borges et al., 2016) have not found significant differences in pregnancy or implantation rates when comparing cultures with and without medium renewal. Similarly, our result showed no significantly differences betweenstudiedgroupsregardingaverageofembryotransferred,chemical\&clinicalpregnancies, implantationRateandlive birth(Rambhia \& Desai, 2014), unable to find significant differences in pregnancy rates between the two methods (with or without medium renewal) have agreed that the blastocyst formation rate from uninterrupted cultures in single medium is significantly higher than that of embryo cultures with medium renewal on day 3,incontrast to our result refreshing significantly improved blastocyst formation from day-3 embryo,signifcantly raises the developed of good quality blastocyst.

(Portella j.et al,2013) compared the development of embryo cultured untill day 5 continously orwith renewal media on day 3 and find that no significant diffirence between two group in blastocyst formation or good quality blastocyst formed,so,preimplantation embryo culture until the fifth in single medium,can be performed without interruption or with renewal at day 3 , not affecting on embryonic development.

(Reed et al., 2009; Vermilyea et al., 2012), have agreed that uninterrupted cultures produce more blastocysts for transfer,in contrast in our study there were significant difference in rate of blastocyst formation in group with medium renewal higher than group without medium renewal.

(Nuno costa-Borges et al,2016) reported the comparison of clinical outcome between of embryo medium renewed at day 3 \&embryo cultured without renewal at day 3 and they find no statically significant differences in implantation or full term development rates were found between two group, this agreed with our data as showed no significant difference between the studied groups regarding number and term among the clinical pregnancy.

Merton et al. (2007) have shown the importance of air quality and have suggested that the accumulation of VOCs in culture systems may have daramatic results on miscarriage rate.

On other hand when (Nuno costa-Borges et al,2016) compared between singleton and twins percentage from embryo cultured in asingle medium with renewal or without renewal result showed no significant difference between two study groups, this result agreed with our study. 
The design of our study has been improved as a cohort of sibling oocytes had been used. This measure would have allowed comparisons not only between patients, but also between embryos in the same cohort, removing the bias generated by circumstances occurring in the laboratory during the investigations and we find that In day-3 Grade-I embryos, improvement (Change from day-3 grade-II to day-5 grade-I and/or persistence of day-3 grade-I as day-5 grade-I) was significantly more frequent among refreshed group, stable (persistence of day- 3 grade-II as day-5 grade-II and/or persistence of day-3 grade-III as day- 5 grade-III) was non-significantly different among refreshed and non-refreshed groups, while deterioration (Change from day-3 grade-I to day-5 grade-II and/or change from day-3 grade-II to day-5 grade-III and/or change from day-3 grade-III to day-5 arrest) was significantly more frequent among non-refreshed group.

In day-3 Grade-II embryos, improvement (Change from day-3 grade-II to day-5 grade-I and/or persistence of day-3 grade-I as day-5 grade-I) was non-significantly different among refreshed group and non-refreshed group, while stable (persistence of day-3 grade-II as day- 5 grade-II and/or persistence of day-3 grade-III as day- 5 grade-III) was significantly more frequent among refreshed group while deterioration (Change from day- 3 grade-I to day- 5 grade-II and/or change from day-3 grade-II to day-5 grade-III and/or change from day-3 grade-III to day-5 arrest) was significantly more frequent among non-refreshed group.

In day-3 Grade-III embryos, no significant difference between refreshed and non-refreshed media regarding changes.

\section{Conclusions:-}

The results obtained in this study founded statistically significant differences between cultures with and without medium renewal for blastocyst formation \& good quality embryo in refreshed group. Improvement Change from day-3 grade-II to day- 5 grade-I and/or persistence of day-3 grade-I as day- 5 grade-I was significantly more frequent among refreshed group However, a non-significant trend favoring cultures without medium renewal was observed in clinical pregnancy or positive pregnancy rates and gestational age.

\section{References:-}

1. Biggers JD, Racowsky C. The development of fertilized human ova to the blastocyst stage in KSOM (AA) medium: is a two-step protocol necessary?. Reproductive Biomedicine Online 2002;5(2):133-40.

2. Blake DA, Farquhar CM, Johnson N, ProctorM. Cleavage stage versus blastocyst stage embryo transfer in assisted conception. Cochrane Database of Systematic Reviews 2007, Issue 4. [DOI: 10.1002/14651858.CD002118.pub3.

3. Brison DR, Houghton FD, Falconer D, Roberts SA, Hawkhead J, Humpherson PG, Lieberman BA, Leese HJ. Identification of viable embryos in IVF by non-invasive measurement of amino acid turnover. Hum Reprod. 2004;19:2319-2324.

4. Cohen J, Gilligan A, Esposito W, Schimmel T, Dale B. Ambient air and its potential effects on conception in vitro. Hum Reprod 1997;12:1742-9.

5. Costa-Borges, Bellés M, Meseguer M, Galliano D, Ballesteros A, Calderón G. Blastocyst development in single medium with or without renewal on day 3: a prospective cohort study on sibling donor oocytes in a time-lapse incubator. Fertil Steril. 2016;105:707-13.

6. Dumoulin JC, Land JA, Van Montfoort AP, Nelissen EC, Coonen E, Derhaag JG, et al. Effect of in vitro culture of human embryos on birthweight of newborns. Human Reproduction 2010;25(3):605-12.

7. GardnerDK, LaneM.Amino acids and ammonium regulate mouse embryo development in culture. Biol Reprod 1993;48:377-385.

8. Hashimoto S, Nishihara T, Murata Y, Oku H, Nakaoka Y, Fukuda A, et al. Medium without ammonium accumulation supports the developmental competence of human embryos. J Reprod Dev. 2008; 54 (5):370 \pm 4. PMID.

9. Hoelker, M. et al., 2010.Effect of embryo density on in vitro developmental characteristics of bovine preimplantative embryos with respect to micro and macroenvironments. Reproduction in domestic animals $=$ Zuchthygiene, 45(5), pp.e138-45.

10. Jiao GZ, Cui W, Yang R, Lin J, Gong S, Lian HY, et al. Optimized Protocols for In Vitro Maturation of Rat Oocytes Dramatically Improve Their Developmental Competence to a Level Similar to That of Ovulated Oocytes.Cellular reprogramming. 2016; 18(1):17 29 . Epub 2015/12/19.

11. Kleijkers, S.H.M. et al., 2015. Age of G-1 PLUS v5 embryo culture medium is inversely associated with birthweight of the newborn. Human Reproduction, 30(6), pp.1352-1357. 
12. Mantikou E, Youssef MAFM, van Wely M, van der Veen F, Al-inany HG, Repping S, et al. Embryo culture media and IVF/ICSI success rates: A systematic review. Human Reproduction Update 2013;19:210-20.

13. Merton JS, Vermeulen ZL, Otter T, Mullaart E, de Ruigh L, Hasler JF. Carbonactivated gas filtration during in vitro culture increased pregnancy rate following transfer of in vitro-produced bovine embryos. Theriogenology 2007;67:1233-8.

14. Portella J, Noriega--H L, Sepْlveda S. Grupo PRANOR de Reproduccín Asistida, Lima, Per.. Accredited Redlara centerwithout renewal of fresh medium on day 3, in a program of oocyte donationPosters -- 11 st General Congress of the Latin American Network of Assisted Reproduction, Panama City -- May de 2013.

15. Rambhia P, Desai N. Global Medium Is Effective as a Single One-step Medium for Uninterrupted Culture to Blastocyst in the Embryscope: Preliminary Pregnancy and Clinical Outcome Data. Fertil Steril. 2014;101:e29.

16. Reed ML, Hamic A, Thompson DJ, Caperton CL. Continuous uninterrupted single medium culture without medium renewal versus sequential media culture: a sibling embryo study. Fertil Steril. 2009;92:1783-6.

17. Wang J, Sauer MV. In vitro fertilization (IVF): a review of 3 decades of clinical innovation and technological advancement. Therapeutics and clinical risk management. 2006 Dec;2(4):355.

18. Wintner EM, Hershko-Klement A, Tzadikevitch K, Ghetler Y, Gonen O, Wintner O, Shulman A, Wiser A. Does the transfer of a poor quality embryo together with a good quality embryo affect the In Vitro Fertilization (IVF) outcome?. Journal of ovarian research. 2017 Dec;10(1):2.

19. Y.M.Elhassan ${ }^{\mathrm{a}}$ G.Wu $\mathrm{u}^{\mathrm{b}}$ A.C.Leanez ${ }^{\mathrm{a}}$ R.J.Tasca ${ }^{\mathrm{c}}$ A.J.Watson ${ }^{\mathrm{d}}$.E.Westhusin ${ }^{\mathrm{a}}$. Amino acid concentrations in fluids from the bovine oviduct and uterus and in ksom-based culture media, Volume 55, Issue 9, 1 June 2001, Pages 1907-1918.

20. Youssef M, Mantikou E, van Wely M, Van der Veen F, Al-Inany HG, Repping S, Mastenbroek S. Culture media for human pre-implantation embryos in assisted reproductive technology cycles. The Cochrane Library. 2015 Jan 1.

21. Mortimer D, Mortimer S. Quality and Risk Management in the IVF Laboratory, 2nd edn. Cambridge: Cambridge University Press, 2015.

22. Nuno Costa-Borges, Ph.D.,a Marta Bell_es, M.Sc.,b Marcos Meseguer, Ph.D.,c Daniela Galliano, M.D.,b Agustin Ballesteros, M.D.,b and Gloria Calder_on, Ph.D..Blastocyst development in single medium with or without renewal on day 3: a prospective cohort study on sibling donor oocytes in a time-lapse incubator Fertil Steril_2016;105: 707-13._2016 by American Society for Reproductive Medicine.

23. Hiura H., Toyoda M., Okae H., Sakurai M., Miyauchi N., Sato A., Kiyokawa N., Okita H., Miyagawa Y., Akutsu H., et al. (2013). Stability of genomic imprinting in human induced pluripotent stem cells. BMC Genet. 14, 32 10.1186/1471-2156-14-32 .

24. Biggers JD, Summers MC. Choosing a culture medium: making informed choices. Fertil Steril 2008;90:47383.

25. Macklon NS, Pieters MHEC, Hassan MA, Jeucken PHM, Eijkemans MJC, Fauser BCJM. A prospective randomized comparison of sequential versus monoculture systems for in-vitro human blastocyst development. Hum Reprod 2002;17: 2700-5 .

26 Fauque P, Jouannet P, Lesaffre C, Ripoche M, Dandolo L, Vaiman D, et al. Assisted reproductive technology affects developmental kinetics, H19 Imprinting control region methylation and H19 gene expression in individual mouse embryos. BMC Developmental Biology 2007;7:116. [DOI:10.1186/1471-213X-7-116.

27 Vermilyea M, Anthony J, Graham J, Tucker M. Op-3 Clinical Outcomes from an Uninterrupted Culture Medium Protocol. Reprod BioMed Online. 2012;24:S2.

28 Renewal of culture media on day 4 of human ivf embryoDevelopment results in a higher blastulation rate when compared to supplementationof the media. 